bioRxiv preprint doi: https://doi.org/10.1101/2022.01.27.477556; this version posted January 28, 2022. The copyright holder for this preprint (which was not certified by peer review) is the author/funder, who has granted bioRxiv a license to display the preprint in perpetuity. It is made available under aCC-BY-NC-ND 4.0 International license.

\title{
Protein Refolding Guided by High-Throughput Differential Scanning Fluorimetry: a case study of an
} HtrA-Family Bacterial Protease

Michael Ronzetti ${ }^{1,2}$, Bolormaa Baljinnyam ${ }^{1}$, Utpal Pal ${ }^{2}$, Anton Simeonov ${ }^{1 *}$

${ }^{1}$ National Center for Advancing Translational Sciences, 9800 Medical Center Drive, Rockville, MD 20850

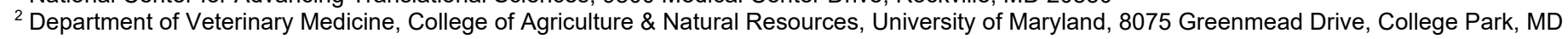
20740

*Corresponding Author: asimeono@nih.gov

\section{Highlights}

- Bacterial HtrA proteins represent an attractive target for next-generation antibiotics discovery.

- Natively purified HtrA proteins coelute with activating peptides and other contaminants, shifting the tertiary and quaternary structure and impacting activation of the protein.

- A denaturing purification of HtrA from inclusion bodies offers substantially improved yields while stripping protein of these contaminating species.

- Our extended screening method for optimal refolding conditions using a commercially available buffer screen and high-throughput differential scanning fluorimetry assay allowed us to generate HtrA protein with high purity and

\section{Abstract} high yield.

The high temperature requirement A ( $\mathrm{HtrA}$ ) serine protease family presents an attractive target class with recent interest for antibacterial development. These proteins contain numerous binding sites and regulatory loops and display diverse oligomerization patterns that depend on substrate occupancy and type. HtrA proteins that are purified natively often coelute with peptides and activating species, shifting oligomerization and protein structure to different activated populations. Here, a redesigned approach to HtrA production by purifying the protein from inclusion bodies under denaturing conditions, and screening for optimal refolding buffer composition using biophysical techniques that are function-agnostic and don't rely on any target-specific readouts or assays is described. This systematic workflow will translate to the production of HtrA-family proteins in higher quantities and of purer composition than the current literature standard.

\section{Subject Category}

Protein purification methods

\section{Keywords}

Protein refolding, protein purification, buffer screening, high-throughput screening, differential scanning fluorimetry, HtrA

\section{Graphical Abstract}

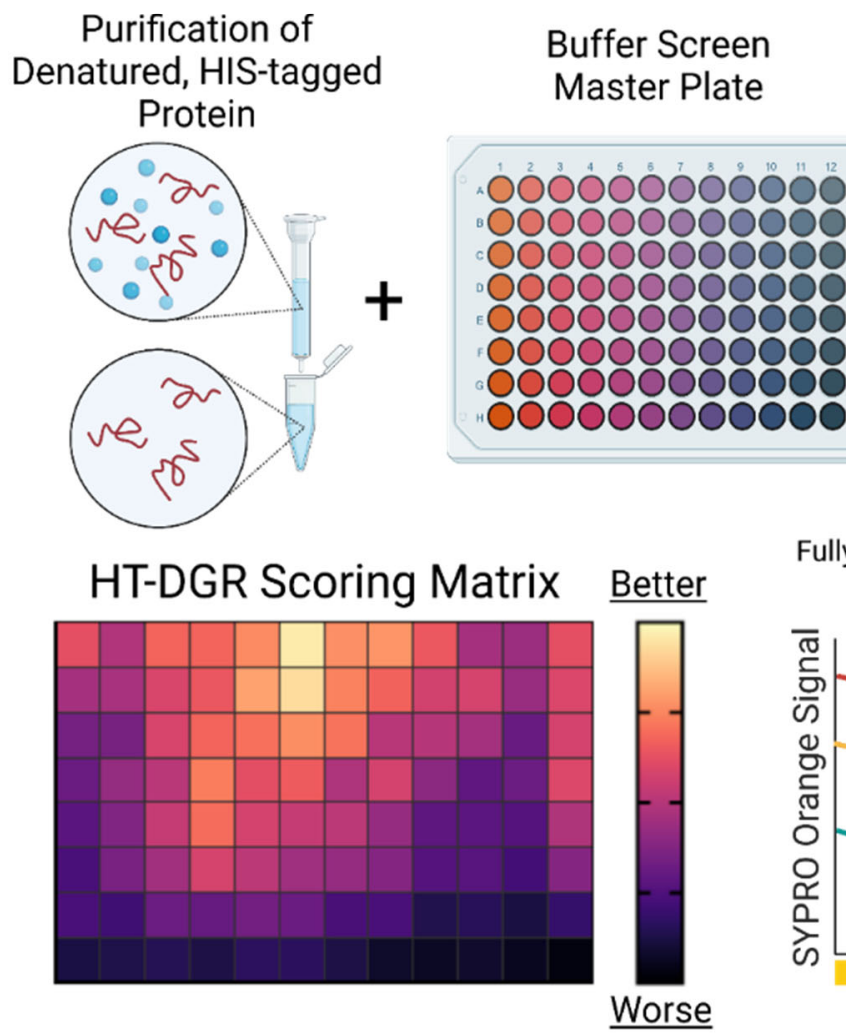

Buffer Screen
HT-DGR

Plate
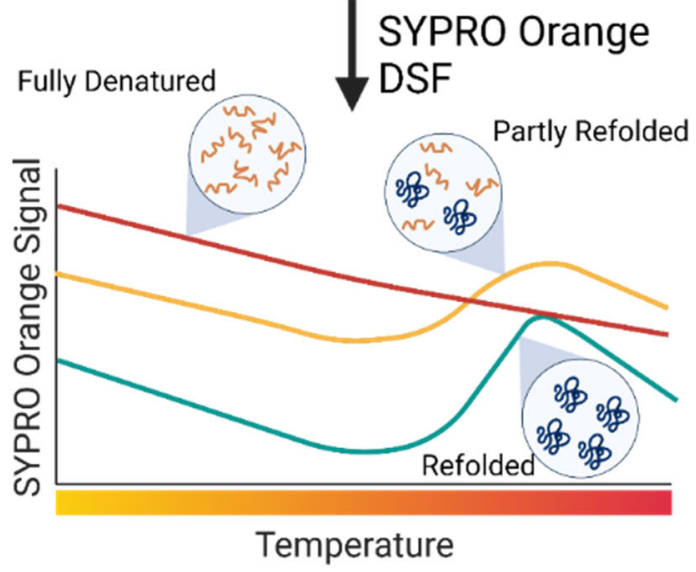
bioRxiv preprint doi: https://doi.org/10.1101/2022.01.27.477556; this version posted January 28, 2022. The copyright holder for this preprint (which was not certified by peer review) is the author/funder, who has granted bioRxiv a license to display the preprint in perpetuity. It is made available under aCC-BY-NC-ND 4.0 International license.
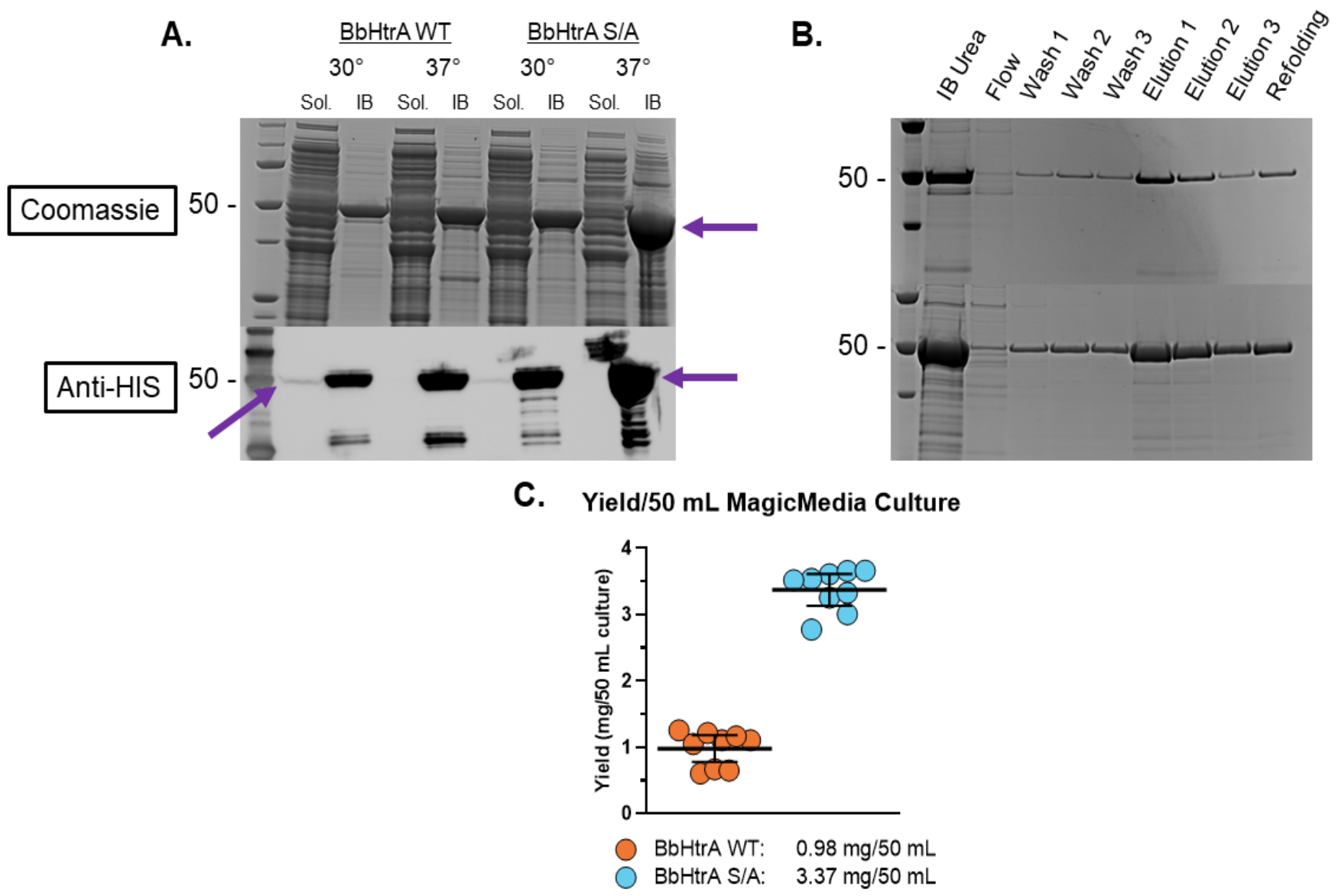

Figure 1 (A) Expression levels of BbHtrA protein in soluble vs. IB fractions of a $2 \mathrm{~mL}$ small-scale culture at different temperatures using the MagicMedia autoinduction system, tested by SDS-PAGE and immunoblotting. BbHtrA has an expected molecular weight of $\sim 50 \mathrm{kDa}$ (purple arrows). (B) Proof-of-concept workflow of a denaturing purification and refolding of BbHtrA proteins in PBS results in a highly pure protein. (C) The protein amount after purification and refolding was measured using the Bradford assay. Expression yields for BbHtrA WT and S/A reach $0.98 \mathrm{mg} / 50 \mathrm{~mL}(0.78-1.18 \mathrm{mg} / 50$ $\mathrm{mL} 95 \% \mathrm{Cl})$ and $3.37 \mathrm{mg} / 50 \mathrm{~mL}(3.13-3.61 \mathrm{mg} / 50 \mathrm{~mL} 95 \% \mathrm{Cl})$ when testing 9 separate $2 \mathrm{~mL}$ small-scale cultures at $37^{\circ} \mathrm{C}$ for 24 hours.

Proteases from the high temperature requirement $A$ family are widely expressed across the animal kingdom and essential to the survival and infectivity of bacteria and other microbes of concern to public health, making them particularly tractable as next-generation antibiotic targets for drug discovery campaigns [1, 2]. HtrA homologs display a wide range of quaternary structures that vary in oligomerization and shape, and the majority of HtrA protein structures remain unstudied [3-9]. All known bacterial HtrA-family proteins form homo-oligomers that shift to higher-order oligomeric structures by different signals, most often by allosteric regulations through the PDZ domains [7, 10-14]. When a substrate binds to the regulatory PDZ domain, it causes conformational changes in the regulatory loops of the protease domain, removing blocks from the oxyanion hole and the $\mathrm{S} 1$ specificity pocket and allowing for proteolytic or holdase-like chaperone activity [4]. Previous groups have reported the presence of contaminating peptides that co-purify with and influence the oligomerization of HtrA [4]. Following up, Bai et al. directly show copurified DegQ substrates by mass spectrometry after native purification from E. coli culture [15]. Purifying HtrA proteins under denaturing conditions would provide a means of removing any potential $\mathrm{HtrA}$-bound ligands. The $B$. burgdorferi $\mathrm{HtrA}, \mathrm{BbHtrA}$, is a therapeutic target shown to contribute to borrelial dissemination in the host and the pathogenesis of Lyme disease [16-19]. This manuscript presents a roadmap and protocols for an improved overexpression and denaturing purification of BbHtrA followed by function-agnostic biophysical screening for the optimal refolding conditions.

\section{Cloning and expression of the HtrA protein}

The BbHtrA amino acid sequence was parsed using the SignalP 3.0 signal peptide detection program with hidden Markov model (HMM) and neural network modes. Interestingly, the HMM model highlights a signal peptide cleavage site between position 28 and 29, while the neural network mirrors prior literature in detecting a peptide cleavage site between positions 37 and 38 and was chosen moving forward [16]. The BbHtrA gene strand (AAC66500, 1326 bp) was cloned into the $\mathrm{pET} 28 \mathrm{a}(+)-\mathrm{TEV}$ vector using the $\mathrm{Ndel} / \mathrm{BamHI}$ restriction sites, retaining the $\mathrm{N}$-terminal $6 \mathrm{XHIS}$ tag and TEV protease site for downstream tag cleavage. These constructs were verified at the T7 promoter for correct insertion, after which BbHtrA WT and the inactive S226A point mutant (S/A) were transformed into BL21 Star (DE3) chemically-competent $E$. coli which contain a rne131 mutation that leads to higher mRNA stability and thus higher-level protein expression over 
standard BL21 strains. For the transformation reaction, $10 \mathrm{ng}$ of plasmid in $5 \mu \mathrm{L} \mathrm{ddH}_{2} \mathrm{O}$ was mixed gently with one vial of BL21 Star cells and transformed following the manufacturer's standard protocol. $80 \mu \mathrm{L}$ of the reaction was streaked onto kanamycin agar plates and incubated at $37^{\circ} \mathrm{C}$ overnight, after which the protein expression of three separate colonies was checked with a small-scale culture according to the manufacturer's protocol $\left(2 \mathrm{~mL}\right.$ culture of Terrific Broth at $37^{\circ} \mathrm{C}$ overnight, $50 \mu \mathrm{g} / \mathrm{mL}$ kanamycin, $0.5 \mathrm{mM}$ IPTG). The culture was spun down $\left(2,000 \mathrm{x} \mathrm{g}, 10 \mathrm{~min}, 4^{\circ} \mathrm{C}\right)$ and lysed using 250 $\mu \mathrm{L}$ BugBuster Master Mix for $30 \mathrm{~min}$ at room temperature (RT). After spinning down the crude lysates $(20,000 \times \mathrm{g}, 30 \mathrm{~min}$, $4{ }^{\circ} \mathrm{C}$ ) and collecting the suspension, $5 \mu \mathrm{L}$ of each lysate $\left(1: 50^{\text {th }}\right.$ of the original culture) was separated using SDS-PAGE and analyzed by immunoblotting for the histidine tag (1:2500 $\alpha-\mathrm{HIS})$. Clones with the highest expression of protein for each mutant were selected to move forward (Fig S1A).

The biomass and BbHtrA yield was further improved by using a high-density autoinduction media, an expression system that is intervention-free after inoculation and improves the yields of target proteins under T7 promoter by using differentially-metabolized carbon sources as compared to traditional multistep LB-IPTG induction [20, 21]. BbHtrA WT and S/A were overexpressed in $2 \mathrm{~mL}$ MagicMedia autoinduction media at both 30 and $37^{\circ} \mathrm{C}$ to monitor protein yield and whether the protein is expressed solubly or not. As observed in prior testing with BbHtrA and other HtrA homologs inhouse, HtrA proteins tend to express most in inclusion bodies (IB) and shift expression towards the soluble fraction when the temperature is lowered at the expense of total yield (Fig 1A). The expression of the S/A mutant was also higher relative to the wildtype protein, though the protein almost entirely appears in the inclusion body fraction (Fig 1A). A pilot denaturing purification was then performed to assess the purity using $0.2 \mathrm{~mL}$ Ni-NTA spin-columns according to manufacturer's protocol and subsequent refolding in PBS (1X, pH 7.4). The resulting preparations of BbHtrA WT and S/A had a purity of $98.9 \%$ and $97.5 \%$, respectively, when analyzed by SDS-PAGE and Coomassie staining (Fig 1B). Testing the target protein yield of 9 separate small-scale cultures (performed in parallel at $37^{\circ} \mathrm{C}$ for 24 hours) with the Bradford assay gave a final yield for BbHtrA WT and S/A at $0.98 \mathrm{mg} / 50 \mathrm{~mL}$ MagicMedia $(0.78-1.18 \mathrm{mg} / 50 \mathrm{~mL} 95 \% \mathrm{Cl})$ and 3.37 $\mathrm{mg} / 50 \mathrm{~mL}$ MagicMedia (3.13 - $3.61 \mathrm{mg} / 50 \mathrm{~mL}$ 95\% Cl) (Fig 1C), respectively.

\section{Screening for optimal refolding conditions}

Purifying functional protein from inclusion bodies presents unique challenges around the proper solubilization and refolding of denatured proteins to their native conformations while producing acceptable yields. Biter et al. established a differential scanning fluorimetry (DSF) -guided workflow to screen buffers for the optimal refolding conditions by diluting purified, denatured protein into a matrix of buffer agents and salt concentrations and then monitoring for the presence and magnitude of thermal melting transitions [22]. This method is especially powerful because the primary readout is agnostic to the target protein's function, providing information on refolding conditions without the requirement for functional testing of your target protein. Here, the utilization of previous DSF-guided refolding workflow is greatly improved by compression into a 384-well format to increase sample throughput and the introduction of a new scoring parameter to better analyze thermal unfolding curves.

To perform the high-throughput DSF-guided refolding (HT-DGR) screen, IBs containing BbHtrA S/A were resuspended in Denaturing/Binding buffer $(20 \mathrm{mM}$ phosphate buffer, $\mathrm{pH} 7.5,5 \mathrm{mM} \mathrm{KCl}, 500 \mathrm{mM} \mathrm{NaCl}, 8 \mathrm{M}$ urea, $20 \mathrm{mM}$ imidazole) at a concentration of $100 \mathrm{mg} \mathrm{IB} / \mathrm{mL}$, shaken at RT for 1 hour, and centrifuged at 20,000 $\mathrm{g} \mathrm{g}$ for $10 \mathrm{~min}$ at RT. The denatured protein was then purified as described earlier using $1.0 \mathrm{~mL}$ Ni-NTA columns. The Hampton Research Solubility and Stability Screen 2 was used as the primary buffer screen because it has a wide-range $\mathrm{pH}$, buffering agent and $\mathrm{NaCl}$ concentration matrix in 96-well format and was used internally with good results in other buffer screening projects (Fig 2A). The recommended dilution of $4 \mathrm{X}$ from the master plate was used to construct the HT-DGR screen (buffering agent final concentration: $50 \mathrm{mM}, \mathrm{NaCl}$ concentration: 0-1000 mM). The 96-well plate was compressed into the four quadrants of a 384-well plate using an Apricot PP-384 pipettor by dispensing $10 \mu \mathrm{L}$ of each well from the master plate into $28 \mu \mathrm{L}$ of water, making a $4 \mathrm{X}$ dilution in a final volume of $40 \mu \mathrm{L}$ (after the subsequent protein dispense). Purified BbHtrA S/A was diluted to $5 \mathrm{mg} / \mathrm{mL}$ in denaturing buffer and $2 \mu \mathrm{L}$ of protein was dispensed into each well of the 384-well plate for a final protein concentration of $0.25 \mathrm{mg} / \mathrm{mL}(5 \mu \mathrm{M})$. The plates were sealed with a foil and placed on a shaker at $100 \mathrm{rpm}$ at $4{ }^{\circ} \mathrm{C}$. Samples taken at 30 - and 90 -minute incubation periods gave low and noisy signal, which prompted an extension to an 18-hour incubation to allow for a more complete refolding. A greater refolding signal is expected from shorter incubation times at higher incubation temperatures as reflected in the Biter et. al protocol, however, higher yields of desired protein refolding are anticipated at lower temperatures due to less protein precipitating through aggregation [2224].

Finally, the DSF assay plate was constructed by dry-spotting $5 \mathrm{~nL}$ of SYPRO Orange with an acoustic dispenser (Echo 555, Labcyte) into a 384-well PCR plate. The plate was spun down at $200 \times \mathrm{g}$ for 1 min before dispensing $5 \mu \mathrm{L}$ of the refolding reaction per well and mixing using an Apricot PP-384 pipettor. The plate was sealed, spun down, and immediately run on a Roche LightCycler $480 \mathrm{II}$ using standard DSF conditions $\left(20-95^{\circ} \mathrm{C}, 0.13^{\circ} \mathrm{C} /\right.$ second, 4 acquisitions per ${ }^{\circ} \mathrm{C}$ ). The resulting thermal shift data set was then analyzed using Roche Thermal Shift Analysis software to derive

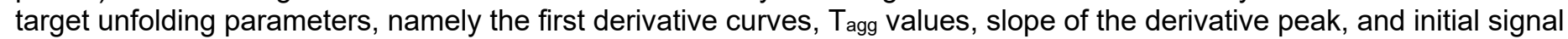
before heating, using standard program parameters.

\section{Analyzing the Thermal Shift Dataset}

Most thermal shift analysis reports unfolding data by determining the midpoint of the sigmoidal fit of thermal unfolding curves as the primary readout for target thermal stability, known as the Tagg. Recent work on DGR assays have 
also compared protein stability by reporting the multiplication of the $\mathrm{T}_{\text {agg }}$ by the height of the first derivative peak, correlating peak height as a measure of the protein refolding yield [22, 24]. In this work, the 'DGR score' is synthesized to describe the refolding stability and yield as represented by the multiplication of the $T_{\text {agg }}$ by the maximum slope of the first derivative peak, and then dividing this result by the initial SYPRO Orange signal before the thermal ramp has been applied. The initial signal of a DSF curve using SYPRO Orange is a convenient indicator of the unfolding status of a sample at baseline; because the dye emits fluorescence energy only after binding to hydrophobic regions in unfolded and aggregated protein species, the initial signal may be assumed to be directly correlated with the amount of unfolded, aggregated, or disordered material present in a refolding reaction.

The results of the BbHtrA S/A HT-DGR screen show reproducible Tagg values across the replicate refolding reactions on our plate, but there is only a small $\sim 1^{\circ} \mathrm{C}$ difference in Tagg among the majority of buffer conditions tested (Fig S1B). The analysis improves after integrating the initial signal and slope of the derivative peak into a "DGR" score, giving the best refolding signals with MES, BisTris, Imidazole, and HEPES buffering agent at pH range 6.0 to 7.5 and $\mathrm{NaCl}$ concentrations below $100 \mathrm{mM}$ (Fig 2B). Indeed, the BbHtrA protein quaternary structure is known to be sensitive to the concentration of salt in buffer, presenting substrate-independent shifts in oligomeric assemblies at different concentrations of $\mathrm{NaCl}$, which fits well with the apparent sensitivity of the refolding process to salt levels [16]. The first derivative of the thermal unfolding curves of the top four selected buffers show single, sharp peaks, unlike less folded or unfolded populations in suboptimal buffer conditions (Fig 2C, green curves are optimal conditons, yellow are suboptimal). The thermal stability of BbHtrA S/A in top refolding conditions was further analyzed using nanoDSF, an orthogonal differential scanning fluorimetry method that monitors shifts in both amino-acid autofluorescence and in backscattering aggregation signals during a thermal ramp $[25,26]$. Following standard nanoDSF experimental design, $5 \mu \mathrm{L}$ of refolded protein was loaded into standard capillaries and onto the capillary tray of a Prometheus NT.48 instrument (Nanotemper) and run using a $5.0^{\circ} \mathrm{C} / \mathrm{min}$ thermal ramp at $50 \%$ LED excitation. Each condition produced strong peaks at $\sim 65^{\circ} \mathrm{C}$, in agreement with $\mathrm{Tm}$ and Tagg measurements, with the MES buffer system chosen to move forward as it produced the sharpest, highest peak in the backscattering aggregation curve (Fig S1C). Notably, an analysis of the HT-DGR screen using only Tagg as the endpoint parameter would advance sodium citrate tribasic [pH 5.0, NaCl $1000 \mathrm{mM}$ ] (Fig S1B, green square) as a top candidate, though the shallow slope and high initial signal in the DSF thermal unfolding curves indicate an unfavorable refolding buffer (Fig 2C, S1D).

The amino acid L-arginine is often included as an additive in refolding buffers to deter aggregation of target proteins by reducing disordered protein-protein interactions along the refolding process [24, 27, 28]. Attempts to integrate L-arginine into the primary buffer screening at high-millimolar concentrations typically used in protein refolding appears to interfere with the SYPRO Orange thermal shift readout, so the alternative nanoDSF technique was applied to samples including L-arginine as described before (Fig S1E). As expected, there was no signal in the negative control water condition. The inclusion of $500 \mathrm{mM}$ arginine in water produced a significant signal, while only minimal signal was apparent when only $\mathrm{NaCl}$ was present (Fig 2D). The MES buffer condition was markedly improved by the addition of $500 \mathrm{mM}$ arginine, which induced a significant shift in the signal span in both intrinsic amino acid fluorescence at $330 \mathrm{~nm}$ (from 32.0 to 55.5) and the backscattering sensor for sample aggregation (from 9.25 to 20.79) (Fig 2D). Notably, while the increase in signal span indicates more properly folded protein contributing to unfolding signals, the presence of arginine did not significantly shift the $T_{\text {agg }}$ or Tonset, suggesting that arginine enhances refolding through a mechanism that does not affect thermal stability of the protein and in agreement with previous work on the role of arginine in protein refolding [27].

As a final test of the MES buffer system with arginine, the refolding reaction was scaled up to $1 \mathrm{mg}$ of total purified BbHtrA S/A diluted to the same concentration as the buffer screen $(0.25 \mathrm{mg} / \mathrm{mL})$ and then dialyzed overnight into the final refolding buffer (50 mM MES, pH 6.5, $50 \mathrm{mM} \mathrm{NaCl}, 500 \mathrm{mM}$ L-arginine). In parallel, native BbHtrA S/A was produced using cell-free protein expression systems following manufacturer's suggested concentrations and protocol with $500 \mathrm{ng}$ plasmid DNA in $50 \mu \mathrm{L}$ reaction volume. Following buffer exchange of the two preparations to PBS, the protein was analyzed using nanoDSF as described earlier. There was no significant difference in the $T_{\text {agg }}$ between cellfree native $\mathrm{BbHtrA}$ and the scaled-up refolded protein, interpreted as final confirmation of an optimal refolding of the BbHtrA protein as a result of HT-DGR-guided buffer design (Fig 2E). Further confirmation of correct BbHtrA refolding was given by the proteolytic cleavage of fluorescent fragments from a casein-BODIPY substrate by wildtype BbHtrA, produced using the above described protocol. Briefly, $50 \mathrm{nM}$ of BbHtrA WT or S/A was mixed with $10 \mu \mathrm{g} / \mathrm{mL}$ casein-BODIPY in a $50 \mu \mathrm{L}$ reaction and immediately read on a Tecan Infinite M1000 in kinetic mode for 20 minutes, where the increase in fluorescence when incubated with BbHtrA WT, but not catalytically-inactive BbHtrA S/A, indicates the proteolytic liberation of fluorescent peptide fragments from the casein-BODIPY substrate (Fig S1F).

Overall, the HT-DGR assay is a widely-accessible method to rapidly screen buffer conditions for optimal refolding of a target protein without dependence on any functional assay. Additionally, by testing conditions from the primary buffer screen with a complementary thermal shift approach, more data is revealed on favorable refolding conditions that would otherwise not be detectable with the SYPRO Orange readout. The flexibility of the HT-DGR format allows researchers to finetune the buffer conditions and concentrations tested with target-dependent changes without interfering with assay design. Finally, a protocol is established for significantly improving the purity and yield of an HtrA-family homolog that will enable more accurate biophysical characterization of these proteins. 
bioRxiv preprint doi: https://doi.org/10.1101/2022.01.27.477556; this version posted January 28, 2022. The copyright holder for this preprint (which was not certified by peer review) is the author/funder, who has granted bioRxiv a license to display the preprint in perpetuity. It is made available under aCC-BY-NC-ND 4.0 International license.

A.

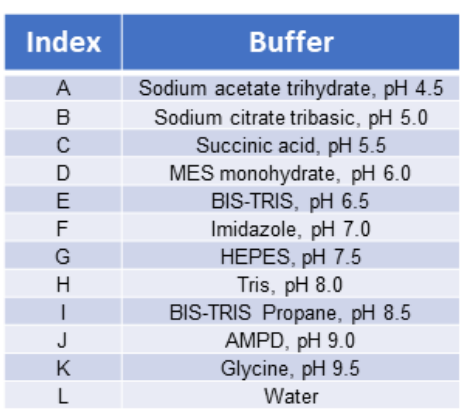

B.

Primary Buffer Screening BbHtrA SIA DGR Score

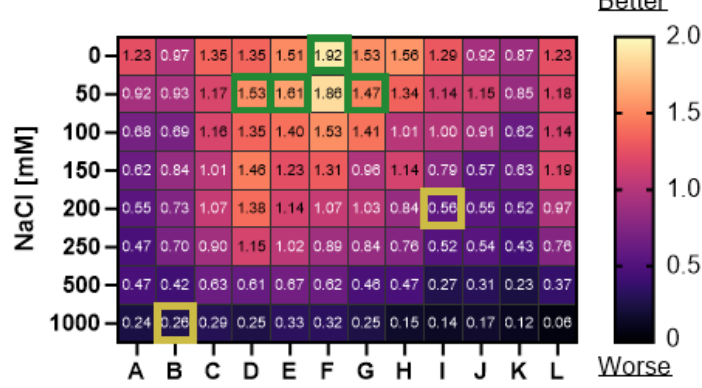

D.

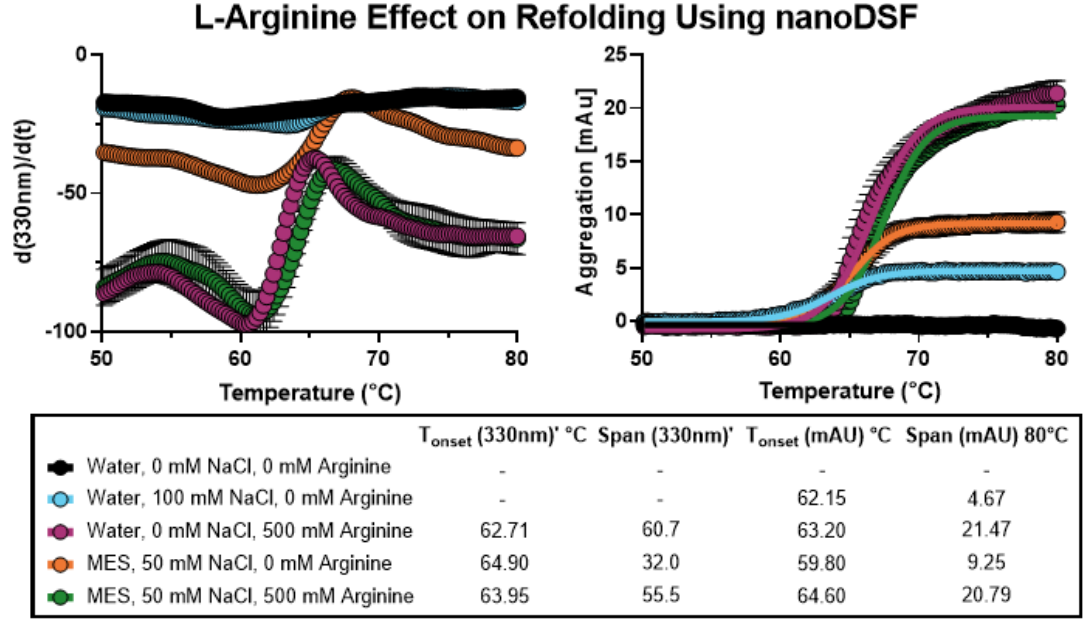

C.

BbHtrA S/A HT-DGR DSF First Derivative of Unfolding Curves

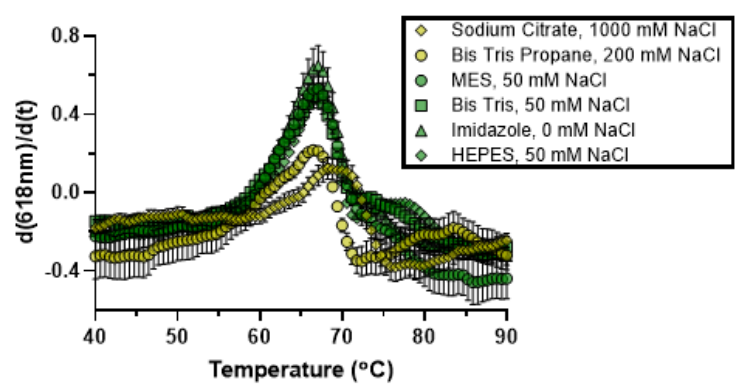

E.

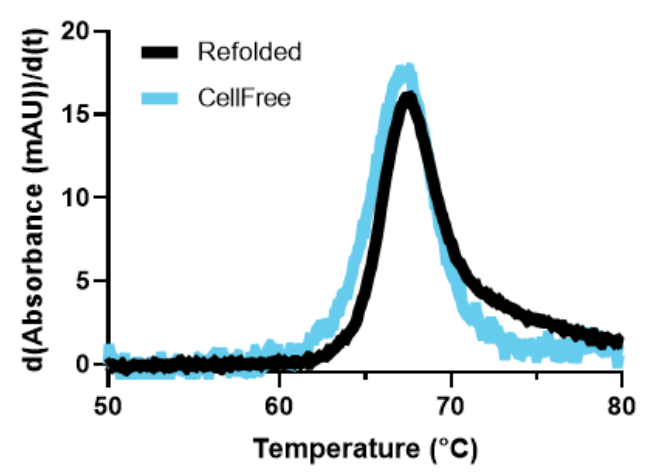

Figure 2 (A) Buffer compositions tested in our HT-DGR screen using the Hampton Research Solubility and Stability Screen 2. These buffers were diluted to a final concentration of $50 \mathrm{mM}$, and paneled in a matrix with 8 concentrations of $\mathrm{NaCl}$ (final 0 - $1000 \mathrm{mM}$ ). (B) DGR scores for BbHtrA S/A show a more optimal folding reaction at lower $\mathrm{NaCl}$ concentrations around $0-100 \mathrm{mM}$ and buffer $\mathrm{pH}$ between $6.0-7.5$. The top four conditions chosen for additive screening are shown with a green box, and representative suboptimal conditions are shown with a yellow box. (C) DSF first derivative unfolding curves for high and low DGR scoring conditions show clear differences in peak height and slope. Note the initial values between different buffering systems indicating different degrees of total unfolded population:

Sodium citrate: $55.79 \pm 8.19$, BisTris Propane: $45.90 \pm 7.75$, MES: $31.58 \pm 6.65$, BisTris: $31.58 \pm 6.73$, Imidazole: $20.87 \pm$ 8.67, HEPES: $33.41 \pm 5.75$. (D) Comparison of the effects of L-arginine on the refolding efficiency of BbHtrA S/A using nanoDSF. The Tonset and span of the $330 \mathrm{~nm}$ fluorescence first derivative and aggregation readouts are given, showing the improvement in refolding yield in the presence of arginine. (E) Comparison of the thermal unfolding profiles of BbHtrA S/A expressed using cellfree native conditions and optimized refolding conditions show similar thermal unfolding profiles.

\section{Author Contribution Statement}

MR: Conceptualization, Methodology, Experimentation, Validation, Analysis, Writing, Review \& Editing BB:

Conceptualization, Methodology, Review \& Editing UP: Conceptualization, Supervision AS: Conceptualization, Methodology, Supervision, Review \& Editing

\section{Funding Source}

This research was supported by the Intramural Research Program of the $\mathrm{NIH}$, National Center for Advancing Translational Sciences (NCATS) (ZIA TR000302-02 to A. Simeonov). 
bioRxiv preprint doi: https://doi.org/10.1101/2022.01.27.477556; this version posted January $28,2022$. The copyright holder for this preprint (which was not certified by peer review) is the author/funder, who has granted bioRxiv a license to display the preprint in perpetuity. It is made available under aCC-BY-NC-ND 4.0 International license.

\section{References}

1. Cho, H., et al., Over-activation of a nonessential bacterial protease DegP as an antibiotic strategy. Commun Biol, 2020. 3(1): p. 547.

2. Backert, S., et al., Extracellular HtrA serine proteases: An emerging new strategy in bacterial pathogenesis. Cell Microbiol, 2018. 20(6): p. e12845.

3. Zhang, L., et al., Cryo-EM structure of Nma111p, a unique HtrA protease composed of two protease domains and four PDZ domains. Cell Res, 2017. 27(4): p. 582-585.

4. Schubert, A., et al., Structures of DegQ from Legionella pneumophila Define Distinct ON and OFF States. J Mol Biol, 2015. 427(17): p. 2840-51.

5. de Regt, A.K., et al., A conserved activation cluster is required for allosteric communication in HtrA-family proteases. Structure, 2015. 23(3): p. 517-526.

6. Thompson, N.J., et al., Substrate occupancy at the onset of oligomeric transitions of DegP. Structure, 2014. 22(2): p. 281-90.

7. Hansen, G. and R. Hilgenfeld, Architecture and regulation of HtrA-family proteins involved in protein quality control and stress response. Cell Mol Life Sci, 2013. 70(5): p. 761-75.

8. Wrase, R., et al., The Legionella HtrA homologue DegQ is a self-compartmentizing protease that forms large 12meric assemblies. Proc Natl Acad Sci U S A, 2011. 108(26): p. 10490-5.

9. Kim, S., R.A. Grant, and R.T. Sauer, Covalent linkage of distinct substrate degrons controls assembly and disassembly of DegP proteolytic cages. Cell, 2011. 145(1): p. 67-78.

10. Krojer, T., et al., Structural basis for the regulated protease and chaperone function of DegP. Nature, 2008. 453(7197): p. 885-90.

11. Jiang, J., et al., Activation of DegP chaperone-protease via formation of large cage-like oligomers upon binding to substrate proteins. Proceedings of the National Academy of Sciences, 2008. 105(33): p. 11939.

12. Clausen, T., C. Southan, and M. Ehrmann, The HtrA Family of Proteases. Molecular Cell, 2002. 10(3): p. 443-455.

13. Pallen, M.J. and B.W. Wren, The HtrA family of serine proteases. Mol Microbiol, 1997. 26(2): p. 209-21.

14. Kolmar, H., P.R. Waller, and R.T. Sauer, The DegP and DegQ periplasmic endoproteases of Escherichia coli: specificity for cleavage sites and substrate conformation. Journal of Bacteriology, 1996. 178(20): p. 5925-5929.

15. Bai, X.C., et al., Characterization of the structure and function of Escherichia coli DegQ as a representative of the DegQ-like proteases of bacterial HtrA family proteins. Structure, 2011. 19(9): p. 1328-37.

16. Russell, T.M., et al., The salt-sensitive structure and zinc inhibition of Borrelia burgdorferi protease BbHtrA. Mol Microbiol, 2016. 99(3): p. 586-96.

17. Ullmann, A.J., et al., Evaluation of Borrelia burgdorferi BbHtrA Protease as a Vaccine Candidate for Lyme Borreliosis in Mice. PLoS One, 2015. 10(6): p. e0128868.

18. Kariu, T., et al., BB0323 and novel virulence determinant BB0238: Borrelia burgdorferi proteins that interact with and stabilize each other and are critical for infectivity. J Infect Dis, 2015. 211(3): p. 462-71.

19. Kariu, T., et al., Proteolysis of BBO323 results in two polypeptides that impact physiologic and infectious phenotypes in Borrelia burgdorferi. Mol Microbiol, 2013. 88(3): p. 510-22.

20. Li, Z., et al., Simple defined autoinduction medium for high-level recombinant protein production using T7-based Escherichia coli expression systems. Appl Microbiol Biotechnol, 2011. 91(4): p. 1203-13.

21. Fox, B.G. and P.G. Blommel, Autoinduction of protein expression. Current protocols in protein science, 2009. Chapter 5: p. Unit-5.23.

22. Amadeo, B.B., et al., DSF Guided Refolding As A Novel Method Of Protein Production. Scientific Reports, 2016.

23. Xie, Y. and D.B. Wetlaufer, Control of aggregation in protein refolding: the temperature-leap tactic. Protein science : a publication of the Protein Society, 1996. 5(3): p. 517-523.

24. Yuanze, W., et al., A Systematic Protein Refolding Screen Method using the DGR Approach Reveals that Time and Secondary TSA are Essential Variables. Scientific Reports, 2017.

25. Magnusson, A.O., et al., nanoDSF as screening tool for enzyme libraries and biotechnology development. FEBS J, 2019. 286(1): p. 184-204.

26. Krakowiak, J., M. Krajewska, and J. Wawer, Monitoring of lysozyme thermal denaturation by volumetric measurements and nanoDSF technique in the presence of N-butylurea. J Biol Phys, 2019. 45(2): p. 161-172.

27. Baynes, B.M., D.I.C. Wang, and B.L. Trout, Role of Arginine in the Stabilization of Proteins against Aggregation. Biochemistry, 2005. 44(12): p. 4919-4925. 
bioRxiv preprint doi: https://doi.org/10.1101/2022.01.27.477556; this version posted January 28, 2022. The copyright holder for this preprint (which was not certified by peer review) is the author/funder, who has granted bioRxiv a license to display the preprint in perpetuity. It is made available under aCC-BY-NC-ND 4.0 International license.

28. François, B., B. François, and M. Mirna, Recombinant protein folding and misfolding in Escherichia coli. Nature Biotechnology, 2004. 


\section{Supplementary}

A.

BbHtrAWT

BbHtrA S/A

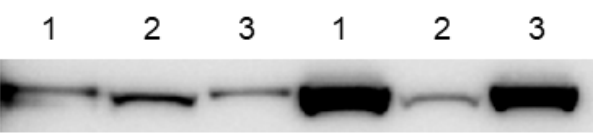

D.

Comparison of MES and Sodium Citrate Refolding Conditions

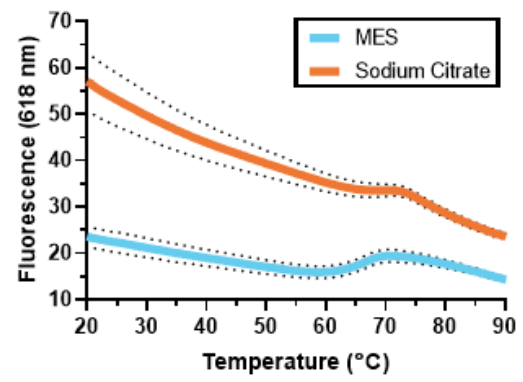

B.

BbHtrA S/A Refolding Screen $T_{\text {agg }}$ Values

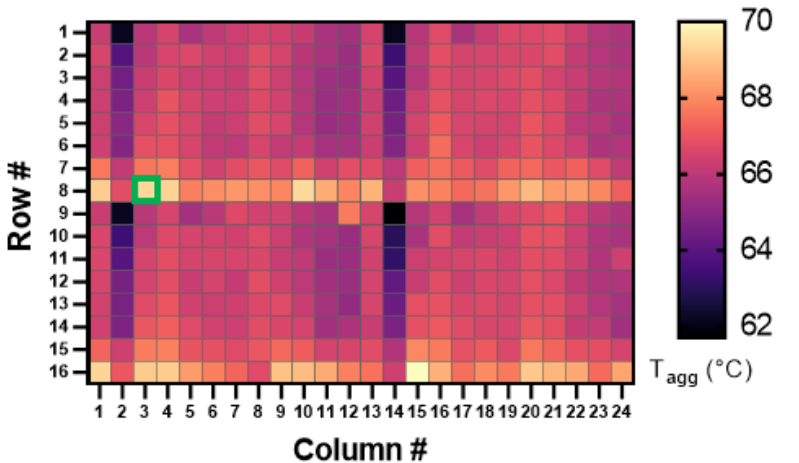

E.

Effect of Arginine in MES Buffer for BbHtrA S/A Refolding Efficiency

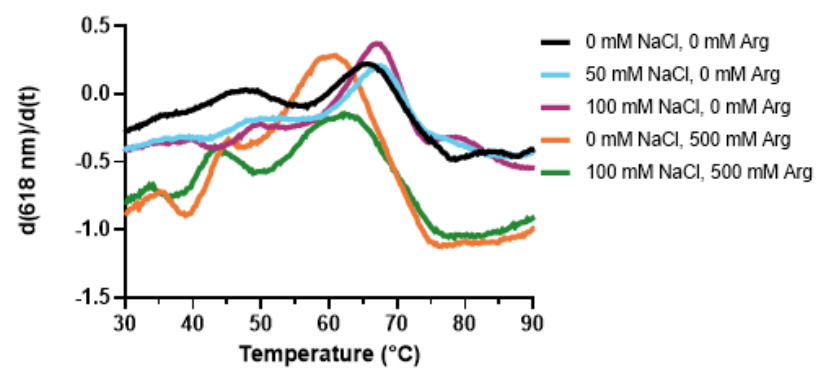

C.

nanoDSF Confirmation of Top Conditions

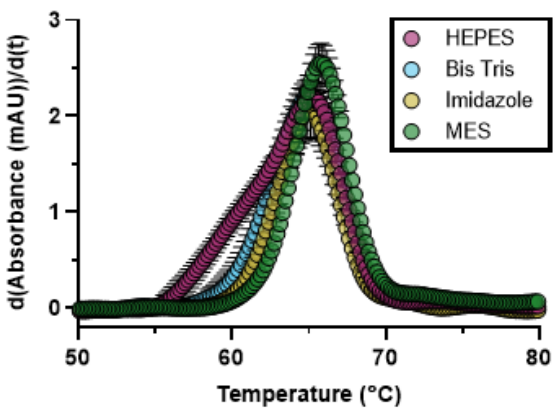

F .

BbHtrA Protease Activity After Refolding

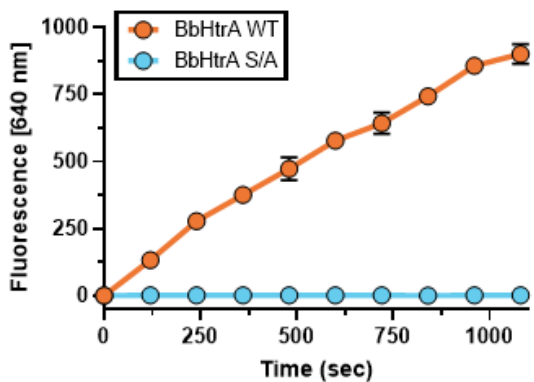

Supporting Figure 1 (A) Immunoblot using anti-HIS antibody against three transformation reactions of BbHtrA WT and BbHtrA S/A pET28a constructs. (B) Heatmap of Tagg values from an HT-DGR screen for the refolding of BbHtrA S/A. The green box is around the sodium citrate tribasic $[\mathrm{pH} 5.0, \mathrm{NaCl} 1000 \mathrm{mM}$ ] condition. (C) The first derivatives of the backscattering curves for the top four conditions using nanoDSF. Data shown is the mean of three replicate biological reactions with error bars showing standard deviations. (D) The first derivatives of the backscattering curves for the top condition from the HT-DGR screen (MES) and the top condition when analyzed using Tagg value only (sodium citrate). Data shown is the mean of three replicate biological reactions with error bars showing standard deviations. (E) The effect of arginine on the refolding of BbHtrA S/A is analyzed using the first derivatives of the DSF thermal unfolding curves for the top refolding buffer condition. Data shown is the mean of three separate biological reactions. (F) The protease activity of BbHtrA WT and S/A after refolding was tested using a caseinBODIPY substrate. Data shown are three independent biological replicates, with error bars showing standard deviations.

Materials

- $\quad$ OneShot BL21 Star (DE3) Chemically Competent Bacteria (Invitrogen \#C601003)

Kanamycin (Millepore Sigma \#K1637-1G)

IPTG (Millepore Sigma \#5810-5GM)

PBS 10X (Millepore Sigma \#6505-OP)

L-Arginine (Millepore Sigma \#A5006)

$\mathrm{NaCl}$ (Millepore Sigma \#S9888)

Ni-NTA spin columns (ThermoFisher $0.2 \mathrm{~mL}$ :\#88224 $1.0 \mathrm{~mL}$ :\#88225)

Pierce Coomassie Plus (Bradford) Assay Reagent (ThermoFisher \#23238)

Imperial Protein Stain (ThermoFisher \#24615)

SYPRO Orange $(5,000 \mathrm{X}$ stock in DMSO, ThermoFisher \#S6650)

Solubility and Stability Screen 2 (Hampton Research \#HR2-413)

384-well white PCR plate (Roche \#04729749001)

384-well nonbinding reaction plate (Corning \#3965) 
bioRxiv preprint doi: https://doi.org/10.1101/2022.01.27.477556; this version posted January 28,2022 . The copyright holder for this preprint (which was not certified by peer review) is the author/funder, who has granted bioRxiv a license to display the preprint in perpetuity. It is made available under aCC-BY-NC-ND 4.0 International license.

$263 \quad$ a-HIS antibody (Cell Signaling Technology \#2365)

264

- $\quad$ nanoDSF standard capillaries (Nanotemper \#PR-C002)

265

NEBExpress CellFree Protein Expression Kit (New England Biolabs \#E5360L) 